\title{
Hipertensão arterial secundária no adulto jovem: um caso clínico
}

Sandra Oliveira, Ana Marta Neves, Teresa Tomaz, Margarida Lima, Francisco Fachado

\begin{abstract}
RESUMO
Introdução: A hipertensão arterial (HTA) secundária corresponde a cerca de 5-10\% dos casos de hipertensão. Perante uma hipertensão em adultos jovens deve-se suspeitar de causas secundárias e proceder a uma investigação clínica, laboratorial e imagiológica adequada. Em adultos jovens (19-39 anos), as causas mais frequentes de HTA secundária são as disfunções tiroideias, a displasia fibromuscular da artéria renal e as doenças do parênquima renal. Relata-se um caso clínico de uma jovem, na qual o estudo de hipertensão secundária revelou hipertiroidismo autoimune.

Descrição do caso: Utente do sexo feminino, 19 anos de idade, que recorreu a consulta de saúde de adultos por palpitações, taquicardia (FC média: 117bpm) e tensão arterial elevada (TA média: 154/86mmHg), objetivadas em ambulatório desde há um mês. Constatou-se ainda ansiedade, insónia inicial, irritabilidade e polifagia. Ao exame objetivo confirmaram-se valores tensionais elevados (TA média: 150/98mmHg) e taquicardia, bem como palpação de bócio difuso, indolor e simétrico, sem nódulos aparentes. Tendo em conta os valores tensionais elevados em dois momentos distintos foi estabelecido o diagnóstico de HTA grau I, tendo a utente sido medicada com propranolol. Do estudo efetuado confirmou-se hipertiroidismo de etiologia autoimune, anemia microcítica e hipocrómica, aspetos sugestivos de tiroidite na ecografia, bem como bócio difuso com captação aumentada na cintigrafia tiroideia, sugestivo de doença de Graves. A utente foi medicada com metibasol 5mg 12/12h e referenciada para consulta de endocrinologia. Verificou-se melhoria da sintomatologia, estabilização tensional e normalização da função tiroideia.

Comentário: Este caso clínico pretende ilustrar a abordagem diagnóstica e terapêutica da HTA no adulto jovem nos cuidados de saúde primários. O hipertiroidismo é uma causa comum de hipertensão sistólica isolada e o seu diagnóstico e tratamento atempados podem prevenir a emergência de complicações e influenciar o prognóstico. Para tal, é fundamental uma boa articulação entre os cuidados de saúde primários e secundários.
\end{abstract}

Palavras-chave: Hipertensão arterial; Hipertensão secundária; Hipertiroidismo; Doença de Graves; Adulto jovem

\section{INTRODUÇÃO}

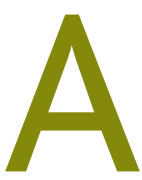

hipertensão arterial (HTA) secundária resulta de uma causa identificável e potencialmente tratável. Os dados epidemiológicos mais recentes apontam para uma prevalência de hipertensão de $26,9 \%$ em Portugal, sendo mais elevada no sexo feminino (29,5\%) do que no sexo masculino (23,9\%). ${ }^{1}$ Não há dados relativos à prevalência da hipertensão secundária em Portugal. Outros estudos apontam para uma prevalência de HTA secundária que varia entre 5-10\% dos casos de HTA..$^{2-4}$ No entanto, dada a elevada prevalência de hipertensão, é provável que seja uma situação frequentemente diagnosticada ao nível dos cuidados de saúde primários, cujo diagnóstico e terapêutica atempadas podem influenciar o prognóstico dos utentes. ${ }^{5-6}$

USF do Minho
As últimas recomendações da European Society of Hypertension/European Society of Cardiology $y^{7}$ sugerem que todos os doentes devem ser submetidos a um rastreio simples para formas secundárias de hipertensão, baseado na história clínica, exame físico e achados laboratoriais. Além disso, uma elevação severa da pressão arterial, o início súbito ou agravamento da HTA, a má resposta à terapêutica e lesões de órgão alvo desproporcionais à duração da HTA devem fazer suspeitar de uma hipertensão secundária. ${ }^{7}$ A norma de orientação clínica da Direção-Geral da Saúde acrescenta ainda que deve ser feito um rastreio de causas secundárias de HTA a indivíduos jovens (idade $<40$ anos). Sempre que haja suspeição de HTA secundária preconiza-se o envio a serviço especializado. ${ }^{8}$

As causas de HTA secundária mais frequentes variam consoante a faixa etária. ${ }^{6}$ Em adultos jovens (19- 
39 anos), as causas mais frequentes são as disfunções tiroideias, a displasia fibromuscular da artéria renal e as doenças do parênquima renal. ${ }^{3,6}$

Relata-se um caso de uma utente jovem com valores tensionais elevados identificados em ambulatório, em que o estudo de hipertensão secundária foi compatível com o diagnóstico de hipertiroidismo de etiologia autoimune.

\section{DESCRIÇÃO DO CASO}

Utente do sexo feminino, 19 anos, raça caucasiana, solteira, estudante de curso de Técnico Auxiliar de Saúde, residente em Braga. Pertence a uma família nuclear, em fase VI do Ciclo de vida familiar de Duvall. Trata-se de uma família de baixo risco, segundo as escalas de $\mathrm{Se}$ govia-Dreyer e Garcia-Gonzalez), de classe de Graffar média-baixa (18 pontos) e APGAR familiar altamente funcional (8 pontos). Sem antecedentes pessoais de relevo. Tem antecedentes ginecológicos de menarca aos 11 anos, menstruação regular, mas abundante, e dismenorreia medicada com contracetivo oral desde há quatro anos. Sem antecedentes cirúrgicos, sem hábitos etílicos, de drogas ou tabágicos e sem alergias conhecidas. Os antecedentes familiares estão representados no genograma, bem como a psicofigura de Mitchel (Figura 1).

A utente recorreu, em março de 2016, à Unidade de Saúde Familiar, no contexto de uma consulta programada com a sua médica de família, por tonturas e palpitações ocasionais, tendo objetivado em ambulatório valores de tensão arterial (TA) persistentemente elevados, sobretudo da TA sistólica, e taquicardia (TA média: 154/86mmHg; frequência cardíaca (FC) média: 117bpm) com um mês de evolução. Quando questionada referiu sentir mais ansiedade, irritabilidade e insónia inicial, que

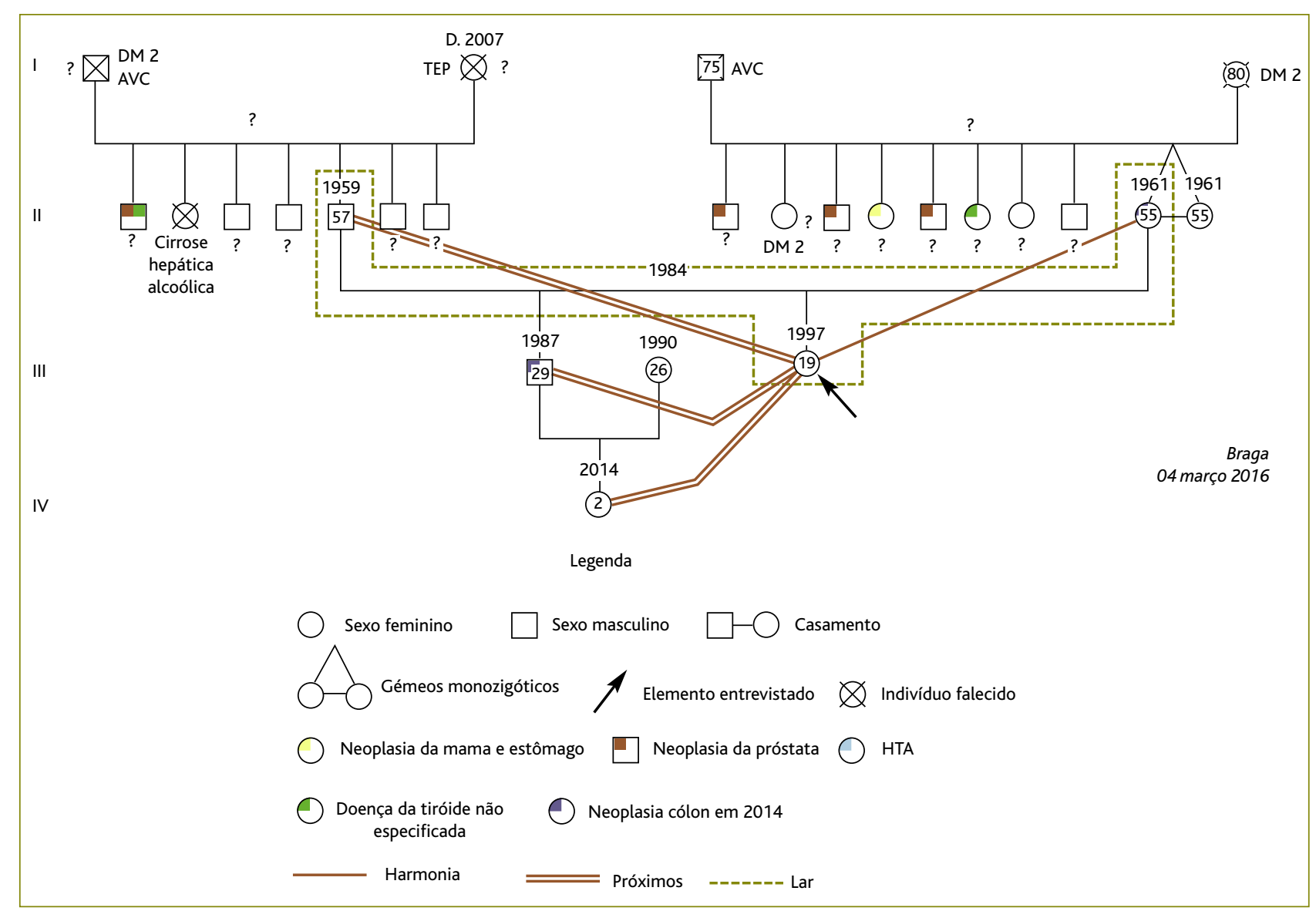

Figura 1. Genograma e psicofigura de Mitchel (Genopro ${ }^{\circledR}$, v. trial).

Legenda: $\mathrm{AVC}=$ acidente vascular cerebral; $\mathrm{DM}=$ diabetes mellitus; $\mathrm{TEP}=$ tromboembolismo pulmonar. 
associou a período de maior stress por ter iniciado recentemente um estágio. Apesar de a doente ter negado outra sintomatologia, a mãe referiu acessos vorazes de polifagia, aparentemente sem repercussão no peso.

Na consulta objetivou-se palidez, peso de $74 \mathrm{~kg}$ (IMC $24,7 \mathrm{~kg} / \mathrm{m}^{2}$ ), perímetro abdominal de $89 \mathrm{~cm}$, TA média de 150/98mmHg (após três medições em ambos os braços), bem como taquicardia (FC média de 114bpm). Uma vez que se constataram valores tensionais elevados em duas ocasiões distintas (valores tensionais superiores a 135/85mmHg em ambulatório e acima de 140/90mmHg no consultório) admitiu-se o diagnóstico de HTA grau I.

Perante o quadro clínico já arrastado iniciou-se terapêutica com propranolol em dose baixa (20 mg/dia) e foi pedido estudo para possíveis causas de hipertensão secundária. A utente foi aconselhada a monitorizar diariamente a tensão arterial em casa e a trazer os registos na próxima consulta.

Um mês depois, a utente mantinha a mesma sintomatologia. Constatou-se ligeira melhoria do perfil tensional e da FC; no entanto, a TA sistólica e a FC permaneciam elevadas (TA média: 145/80mmHg, FC média: $102 \mathrm{bpm})$. Objetivou-se ainda bócio difuso, indolor e simétrico, sem nódulos aparentes - $c f$. os resultados do estudo efetuado no Quadro I. Analiticamente destaca-se uma anemia microcítica e hipocrómica e TSH indoseável, tendo sido solicitado estudo complementar. A dose de propranolol foi aumentada para $40 \mathrm{mg} / \mathrm{dia}$.

Em maio de 2016, a utente referiu melhoria das palpitações e valores tensionais mais estáveis (TA média: 141/65mmHg, FC média: 97bpm). O estudo complementar da anemia foi negativo; no entanto, confirmou-se um hipertiroidismo de etiologia autoimune. Não obstante, a ecografia revelou "tiroide de dimensões e topografia normais, contornos regulares e ecoestrutura globalmente hipoecoica, heterogénea, micronodular, altamente sugestiva de Tiroidite Linfocítica de Hashimoto", achados não concordantes com a clínica nem com o exame físico da utente.

Perante o facto dos anticorpos estimuladores do recetor da TSH (TRABs) não serem comparticipados pelo Serviço Nacional de Saúde, aliado à dissociação clínico-imagiológica, efetuou-se contacto telefónico com o serviço de endocrinologia do hospital da área de referenciação, que aconselhou investigação etiológica pre- viamente ao início do tratamento. Foi discutido com a utente a possibilidade de realizar cintigrafia tiroideia em ambulatório, enquanto aguardaria consulta de endocrinologia, que a utente concordou.

A cintigrafia tiroideia foi concordante com a clínica, demonstrando "bócio difuso com índice de captação muito aumentado, relacionado com hiperfunção muito significativa da glândula, sugestivo de Doença de Graves" (Quadro I). Foi iniciado metibasol 5mg 12/12h e manteve-se o propranolol na dose de $40 \mathrm{mg} / \mathrm{dia}$.

Pelo genograma constatam-se antecedentes familiares de doença da tiroide (não especificada) em dois familiares em $2^{\circ}$ grau, bem como de hipertensão na mãe (com início aos 35 anos) e no irmão (recentemente diagnosticada). Considerando o risco hereditário do hipertiroidismo, o estudo foi alargado a estes familiares, que foi negativo.

Enquanto aguardava consulta hospitalar, a utente foi contactada pela sua médica de família para avaliação intermédia (outubro de 2016) e realização de estudo analítico de controlo, tendo-se objetivado melhoria da sintomatologia - TA: 112/68mmHg, FC: 70bpm e normalização da função tiroideia (Quadro I). Optou-se pela manutenção do metibasol e ajuste de dose do propranolol (20mg/dia). Uma vez atingido o controlo da HTA com medicação dirigida ao hipertiroidismo, a HTA foi considerada como problema inativo.

Em março de 2017, no contexto da primeira consulta de endocrinologia, dada a melhoria sintomática, o bom controlo tensional e da FC, foi suspenso o propranolol e manteve o metibasol na mesma dose. Posteriormente confirmou-se a positividade dos TRABs e o controlo da função tiroideia (Quadro I), tendo diminuído a dose de metibasol (2,5mg/dia).

Atualmente a utente permanece em seguimento em consulta hospitalar.

\section{COMENTÁRIO}

O hipertiroidismo é uma causa comum de hipertensão sistólica isolada que ocorre devido à diminuição da resistência vascular periférica e ao aumento do débito e da frequência cardíaca. ${ }^{9}$ Os estudos não são concordantes em relação ao pico de incidência do hipertiroidismo; no entanto, a maioria aponta para um máximo de incidência na faixa etária dos 20-40 anos. As causas mais frequentes de hipertiroidismo são a doença de 


\begin{tabular}{|c|c|c|c|c|}
\hline Estudo analítico & Março 2016 & Abril 2016 & Outubro 2016 & Março 2017 \\
\hline $\begin{array}{l}\text { Hemoglobina (Hb) } \\
\text { Hematócrito } \\
\text { Volume globular médio } \\
\text { Hb globular média } \\
\text { Concentração de Hb globular média } \\
\text { TSH } \\
\text { Leucócitos } \\
\text { Neutrófilos } \\
\text { Eosinófilos } \\
\text { Basófilos } \\
\text { Linfócitos } \\
\text { Monócitos } \\
\text { Plaquetas } \\
\text { Rdw } \\
\text { VS } \\
\text { Colesterol total } \\
\text { LDL } \\
\text { HDL } \\
\text { Triglicerídeos } \\
\text { Glicose } \\
\text { Creatinina } \\
\text { Na } \\
\text { K } \\
\text { Cl } \\
\text { Sedimento urinário } \\
\text { Ferro } \\
\text { Ferritina } \\
\text { CTFF } \\
\text { Transferrina } \\
\text { Eletroforese Hb } \\
\text { Atc anti-TG } \\
\text { Atc anti-TPO } \\
\text { TRABs } \\
\text { T4 livre } \\
\text { T3 livre } \\
\text { Bilirrubina total } \\
\text { Bilirrubina direta } \\
\text { Fosfatase alcalina } \\
\text { AST } \\
\text { ALT } \\
\text { GGT }\end{array}$ & $\begin{array}{l}11,2 \mathrm{~g} / \mathrm{dL} \downarrow \\
35,4 \% \\
74,2 \mathrm{fL} \downarrow \\
23,6 \mathrm{pg} \downarrow \\
31,7 \mathrm{~g} / \mathrm{dL} \downarrow \\
<0,02 \downarrow \downarrow \\
4,76 \times 10^{3} / \mathrm{mm}^{3} \\
33,9 \% \\
7,5 \% \\
0,2 \% \\
51,1 \% \\
7,3 \% \\
221 \times 10^{3} / \mathrm{mm}^{3} \\
16,1 \% \\
39 \mathrm{~mm} / \mathrm{h} \uparrow \\
123 \mathrm{mg} / \mathrm{dL} \\
53,8 \mathrm{mg} / \mathrm{dL} \\
47 \mathrm{mg} / \mathrm{dL} \\
111 \mathrm{mg} / \mathrm{dL} \\
90 \mathrm{mg} / \mathrm{dL} \\
0,52 \mathrm{mg} / \mathrm{dL} \\
139 \mathrm{mmol} / \mathrm{L} \\
3,8 \mathrm{mmol} / \mathrm{L} \\
105 \mathrm{mmol} / \mathrm{L} \\
\text { Sem alterações }\end{array}$ & $\begin{array}{l} \\
\\
\\
\\
81 \mu \mathrm{g} / \mathrm{dL} \\
13 \mathrm{ng} / \mathrm{mL} \\
385 \mu \mathrm{g} / \mathrm{dL} \\
308 \mu \mathrm{dg} / \mathrm{dL} \\
\text { Sem alterações } \\
92 \mathrm{UL} / \mathrm{mL} \uparrow \\
1592 \mathrm{UL} / \mathrm{mL} \uparrow \uparrow \\
4.04 \mathrm{ng} / \mathrm{dL} \uparrow \\
16,75 \mathrm{ng} / \mathrm{dL} \uparrow \uparrow \\
0,35 \mathrm{mg} / \mathrm{dL} \\
0,20 \mathrm{mg} / \mathrm{dL} \\
74 \mathrm{U} / \mathrm{L} \\
18 \mathrm{U} / \mathrm{L} \\
17 \mathrm{U} / \mathrm{L} \\
9 \mathrm{U} / \mathrm{L}\end{array}$ & $\begin{array}{l}1.02 \mathrm{ug} / \mathrm{dL}(\mathrm{N}) \\
0,39 \mathrm{~S} / \mathrm{L} \\
0,18 \mathrm{~S} / \mathrm{L} \\
53 \mathrm{U} / \mathrm{L} \\
17 \mathrm{U} / \mathrm{L} \\
10 \mathrm{U} / \mathrm{L} \\
10 \mathrm{U} / \mathrm{L}\end{array}$ & $\begin{array}{l}2,17 \mathrm{U} / \mathrm{L} \uparrow \\
1,16 \mathrm{ng} / \mathrm{dL}(\mathrm{N}) \\
3,16 \mathrm{pg} / \mathrm{ml}(\mathrm{N})\end{array}$ \\
\hline Eletrocardiograma & \multicolumn{4}{|c|}{ Ritmo sinusal. FC normal. Sem alterações. } \\
\hline Ecocardiograma transtorácico & \multicolumn{4}{|l|}{ Sem alterações. } \\
\hline Ecografia renal e suprarrenal & \multicolumn{4}{|l|}{ Sem alterações. } \\
\hline Ecografia da tiroide & \multicolumn{4}{|c|}{$\begin{array}{l}\text { Tiroide de dimensões e topografia normais. Contornos regulares e ecoestrutura global- } \\
\text { mente hipoecoica, heterogénea, micronodular, altamente sugestiva de Tiroidite Linfocítica } \\
\text { de Hashimoto. }\end{array}$} \\
\hline Cintigrafia da tiroide & \multicolumn{4}{|c|}{$\begin{array}{l}\text { Bócio difuso com índice de captação muito aumentado, relacionado com hiperfunção } \\
\text { muito significativa da glândula. Estes achados podem estar relacionados com Doença de } \\
\text { Graves. Sem evidentes formações nodulares hiper ou hipofuncionantes. } \\
\text { Índice de captação de pertecnetato pela tiroide: } 30,9 \% \text { (normal: } 1-3,5 \% \text { ). }\end{array}$} \\
\hline
\end{tabular}

Legenda: $\mathrm{Hb}=$ hemoglobina; TSH = hormona estimuladora da tiroide; $\mathrm{Rdw}=$ Red Cell Distribution Width; $\mathrm{VS}=$ velocidade de sedimentação; LDL = lipoproteínas de baixa densidade; $\mathrm{HDL}=$ lipoproteínas de alta densidade; $\mathrm{Na}=$ sódio; $\mathrm{K}=$ potássio; $\mathrm{Cl}=$ cloretos; $\mathrm{CTFF}=$ capacidade total de fixação do ferro; Atc anti-TG = anticorpos anti-tireoglobulina; Atc anti-TPO = anticorpos antitireoperoxidase; $\mathrm{TRABs}=$ anticorpos antirrecetor da TSH; $\mathrm{T} 4=$ tiroxina; $\mathrm{T3}=$ triiodotironina; $\mathrm{AST}=$ aspartato aminotransferase; $\mathrm{ALT}=$ alanina aminotransferase; GGT = Gama GT; $\uparrow$ aumentado, $\downarrow$ diminuído. 
Graves (60-88\%), seguida do bócio multinodular tóxico (8-25\%) e do nódulo tóxico (4-15\%), sendo o sexo feminino o mais atingido. ${ }^{10} \mathrm{~A}$ doença de Gravesé uma patologia autoimune, em que os linfócitos $\mathrm{T}$ são sensíveis aos antigénios tiroideus e estimulam os linfócitos B a produzirem anticorpos contra estes antigénios, nomeadamente os TRABs. Uma vez que nesta patologia existe uma predisposição familiar (cerca de $15 \%$ dos doentes têm um familiar com a mesma doença), ${ }^{10}$ é importante a colheita de uma história familiar detalhada.

A apresentação clínica mais frequente do hipertiroidismo inclui palpitações, ansiedade, aumento da sudorese, diarreia, intolerância ao calor, insónia e, habitualmente, perda ponderal com apetite normal ou aumentado. No entanto, a repercussão no peso pode não ser notória devido à polifagia, como no caso apresentado. As manifestações mais típicas são a taquicardia, arritmias, tremores e aumento da TA sistólica. Os casos mais raros podem cursar com exoftalmia e dermopatia, sobretudo na doença de Graves, ${ }^{9-11}$ bem como com envolvimento respiratório, neurológico, neuromuscular, hematopoiético ou ao nível do sistema reprodutivo. ${ }^{10}$

No caso clínico descrito, a utente apresentava uma anemia ligeira microcítica e hipocrómica, que poderia ser interpretada no contexto das menorragias, pelo que foi solicitado estudo complementar que se revelou negativo. Assim, é mais provável que estes achados sejam explicados pela própria doença de Graves. Apesar de ser uma apresentação atípica da doença, a anemia pode surgir em $22 \%$ dos casos de doença de Graves. ${ }^{12}$ Caracteristicamente, a «anemia da doença de Graves» é normo ou, mais comummente, microcítica, com cinética do ferro normal, podendo estar associada a trombo e a leucopenia. ${ }^{12} \mathrm{~A}$ patogénese permanece desconhecida; no entanto, pensa-se que pode estar relacionada com o efeito do excesso das hormonas da tiroide na eritropoiese que, apesar de estar aumentada, é ineficaz. É expectável a normalização do VGM e da concentração de hemoglobina com o tratamento da doença de Graves. ${ }^{12}$

O diagnóstico de hipertiroidismo passa pelo doseamento da TSH (baixa ou indoseável) eT4 livre (fração metabolicamente ativa) ou T3 livre aumentada. De referir que a toma de anovulatórios pode simular um hipertiroidismo pelo aumento da T3 e T4 totais, pelo que deve ser usado para diagnóstico o doseamento da T3 e T4 livres. ${ }^{10}$ Além disso, a toma oral de estrogénios na dose de 30-35mcg pode ser também uma causa de hipertensão arterial; ${ }^{5}$ no entanto, neste caso seria pouco provável uma vez que a utente já toma o anticoncecional há vários anos e não explicaria todo o quadro clínico.

A ecografia tiroideia permite documentar uma tiroidite e o diagnóstico diferencial com bócio nodular tóxico, que requer outro tipo de abordagem e tratamento. A positividade dos anticorpos antitiroideus documenta a etiologia autoimune da doença, podendo estar elevada na doença de Graves, tiroidite de Hashimoto, bem como na tiroidite subaguda. ${ }^{11}$ Os anticorpos anti-TPO (antitireoperoxidase) são os que se relacionam mais com a disfunção clínica da tiroide e a sua presença está fortemente associada a inflamação linfocítica e lesão glandular. Geralmente permanecem positivos mais tempo e são usados para definir a etiologia autoimune da doença. ${ }^{13} \mathrm{Os}$ anticorpos anti-TG (antitireoglobulina) são menos frequentes e o seu papel é menos claro. Os TRABs, apesar de não serem patognomónicos de doença de Graves, uma vez que podem estar presentes também noutras tiroidites autoimunes onde são anticorpos bloqueadores (e.g., 10\% dos casos de tiroidite de Hashimoto), ${ }^{13}$ são altamente sugestivos da primeira (sensibilidade $>90 \%$ ). ${ }^{11}$ Além disso, os TRABs relacionam-se positivamente com a gravidade da doença e permitem uma monitorização da terapêutica, uma vez que níveis normais no final do tratamento indicam maior probabilidade de remissão. ${ }^{14}$ Embora um resultado positivo não revele se se tratam de anticorpos estimuladores ou bloqueadores, aceita-se concluir que a sua presença num indivíduo com hipertiroidismo seja diagnóstico de doença de Graves..$^{15}$

No caso clínico apresentado, a ecografia tiroideia suscitou dúvidas na etiologia do hipertiroidismo, indicando uma tiroide de dimensões normais e sugerindo a hipótese "altamente sugestiva de Tiroidite Linfocítica de Hashimoto". Este resultado não é concordante com os achados clínicos de hipertiroidismo, nem com a presença de bócio ao exame objetivo ou com os resultados analíticos (nomeadamente a elevação mais notória da T3 livre comparativamente à T4 livre, que é mais sugestiva de doença de Graves). Pela clínica e exame objetivo apresentados seria expectável uma ecografia compatível com tiroidite e tiroide aumentada de volume, a favor de doença de Graves. Estes dados incongruentes podem ser explicados pela base etiopatogénica comum entre a tiroidite de Hashimoto e a doença 
de Graves, ambas doenças autoimunes que se podem manifestar por um largo espetro de alterações clínicas e laboratoriais, variando de hipo a hipertiroidismo no mesmo doente e podendo evoluir de uma para a outra. A primeira, ou tiroidite autoimune crónica, é a causa mais frequente de bócio e hipotiroidismo. Apesar de poder haver alternância entre hiper e hipotiroidismo, é muito rara. Clinicamente manifesta-se por um bócio firme, simétrico e indolor, anti-TPO fortemente positivos em $90 \%$ casos, anti-TG positivos em $20-50 \%$ dos utentes, TSH normal ou aumentada (muito raramente está diminuída). Ecograficamente, a tiroide apresenta um padrão heterogéneo de predomínio hipoecogénico, com istmo espessado. ${ }^{13}$ Por outro lado, a doença de Graves cursa com clínica de hipertiroidismo e bócio difuso e, mais raramente, com oftalmopatia e dermopatia que, quando presentes, são suficientes para confirmar o diagnóstico, ${ }^{15}$ o que, pela sua ausência, dificultou o diagnóstico no caso apresentado.

Outra dificuldade no diagnóstico foi a ausência de comparticipação pelo Serviço Nacional de Saúde do doseamento dos TRABs. Neste caso, após discussão com a utente e com a endocrinologista, optou-se pela realização da cintigrafia tiroideia em ambulatório, o que permitiu esclarecer as dúvidas diagnósticas e o início do tratamento precoce. A cintigrafia tiroideia está indicada para esclarecimento etiológico e diagnóstico diferencial entre doença de Graves, bócio nodular tóxico ou tiroidite subaguda, que requerem diferentes abordagens terapêuticas. Na doença de Graves constata-se um aumento difuso da captação de iodo, ${ }^{11,15}$ como se confirmou no caso descrito, no bócio nodular tóxico hiperfuncionante uma captação localizada de iodo e na tiroidite subaguda habitualmente não se verifica aumento da captação de iodo, uma vez que não ocorre aumento da síntese das hormonas tiroideias - apenas um aumento da sua libertação e o tratamento é apenas sintomático. ${ }^{16}$

As potenciais consequências do hipertiroidismo não tratado são as arritmias (nomeadamente fibrilhação auricular), hipertensão pulmonar, hipertrofia do ventrículo esquerdo e insuficiência cardíaca. ${ }^{9}$

O tratamento da doença de Graves, em primeira linha, passa pelo recurso a fármacos antitiroideus (preferencialmente metibasol $5 \mathrm{mg}$ - exceto no primeiro trimestre da gravidez - no máximo até $60 \mathrm{mg} /$ dia, dependendo da gravidade da doença) ${ }^{10} \mathrm{e}$ beta-bloqueantes para contro- lo dos sintomas adrenérgicos (e.g., propranolol, atenolol, bisoprolol) em utentes com FC em repouso $>$ 90bpm ou com doença cardiovascular coexistente. ${ }^{11,14} \mathrm{O}$ utente deve ser alertado para possíveis efeitos adversos da medicação, como rash pruriginoso, icterícia, fezes acólicas, urina escura, artralgias, dor abdominal, náuseas, fadiga, febre ou faringite. ${ }^{14}$ Além disso, é aconselhável obter-se um hemograma completo e função hepática prévios à introdução do tratamento pelo risco de agranulocitose e dano hepático. ${ }^{17}$ Por rotina, não está recomendado o doseamento da fórmula leucocitária no follow-up. ${ }^{14} \mathrm{Em}$ casos refratários pode ser necessário tratamento com iodo radioativo ou cirurgia. ${ }^{10}$

Os principais objetivos do tratamento são reduzir a TA sistólica, a FC e o débito cardíaco. ${ }^{9}$ No caso da doença de Graves, o tratamento pode ser mantido durante 12 a 24 meses, ${ }^{10}$ até o doente apresentar função tiroideia normalizada (T4 livre normal - a TSH pode demorar meses a normalizar) e título de TRABs negativo. ${ }^{11} \mathrm{~A}$ taxa de remissão na doença de Graves após terapêutica com antitiroideus é de $30-60 \%$, mas nos jovens pode ser inferior (15 a $30 \%$ ao fim de dois ou mais anos). ${ }^{10}$ De realçar que nunca se pode falar em cura, pois podem ocorrer recidivas. ${ }^{10}$

Com este caso clínico pretende-se alertar para a pesquisa de causas secundárias de hipertensão arterial no adulto jovem ao nível dos cuidados de saúde primários, uma vez que o diagnóstico precoce diminui o risco de complicações e um tratamento atempado permite um bom controlo da doença na maioria das situações. Para tal, é fundamental uma boa articulação entre os cuidados de saúde primários e secundários. Além disso, pretende-se fomentar o espírito crítico na interpretação dos exames auxiliares de diagnóstico, que deve ser sempre enquadrado com a clínica.

\section{REFERÊNCIAS BIBLIOGRÁFICAS}

1. Macedo ME, Ferreira RC. A hipertensão arterial nos cuidados de saúde primários, em Portugal: contributo para o conhecimento epidemiológico da população em 2013. Rev Factores Risco. 2015;(36):47-56.

2. Berglund G, Andersson O, Wilhelmsen L. Prevalence of primary and secondary hypertension: studies in a random population sample. $\mathrm{Br} \mathrm{Med}$ J. 1976;2(6035):554-6.

3. Rudnick KV, Sackett DL, Hirst S, Holmes C. Hypertension in a family practice. Can Med Assoc J. 1977;117(5):492-7.

4. Omura M, Saito J, Yamaguchi K, Kakuta Y, Nishikawa T. Prospective study on the prevalence of secondary hypertension among hypertensive patients visiting a general outpatient clinic in Japan. Hypertens Res. 2004;27(3):193-202. 
5. Onusko E. Diagnosing secondary hypertension. Am Fam Physician. 2003;67(1):67-74.

6. Viera AJ, Neutze DM. Diagnosis of secondary hypertension: an age-based approach. Am Fam Physician. 2010;82(12):1471-8.

7. Mancia G, Fagard R, Narkiewicz K, Redon J, Zanchetti A, Böhm M, et al. 2013 ESH/ESC guidelines for the management of arterial hypertension: the Task Force for the Management of Arterial Hypertension of the European Society of Hypertension (ESH) and of the European Society of Cardiology (ESC). Eur Heart J. 2013;34(28):2159-219.

8. Direção-Geral da Saúde. Abordagem terapêutica da hipertensão arterial: norma nº 026/2011, de 29/09/2011, atualizada a 19/03/2013. Lisboa: DGS; 2013.

9. Prisant LM, Gujral JS, Mulloy AL. Hyperthyroidism: a secondary cause of isolated systolic hypertension. J Clin Hypertens. 2006;8(8):596-9.

10. Galvão-Teles A, Garcia e Costa J, Jorge Z, Dias T. Hipertiroidismo/ /Tireotoxicose [Internet]. Lisboa: Núcleo de Endocrinologia, Diabetes e Obesidade [cited 2016 May 29]. Available from: https://pt.scribd.com/ document/301491766/Hipertiroidismo

11. Esteves C, Neves C. Tirotoxicose. Arq Med. 2013;27 Suppl:16-8.

12. Hegazi MO,Ahmed S. Atypical clinical manifestations of Graves' disease: an analysis in depth. J Thyroid Res. 2012;2012:ID768019.

13. Melo M. Tiroidites autoimunes [Autoimmune thyroiditis]. Acta Med Port. 2006;19(5):387-94. Portuguese

14. Bahn RS, Burch HB, Cooper DS, Garber JR, Greenlee MC, Klein I, et al.
Hyperthyroidism and other causes of thyrotoxicosis: management guidelines of the American Thyroid Association and American Association of Clinical Endocrinologists. Endocr Pract. 2011;17(3):456-520.

15. Neves C, Alves M, Delgado JL, Medina JL. Doença de Graves [Graves' disease]. Arq Med. 2008;22(4/5):137-46. Portuguese

16. Intenzo CM, Capuzzi DM, Jabbour S, Kim SM, dePapp AE. Scintigraphic features of autoimmune thyroiditis. Radiographics. 2001;21(4):957-64.

17. Burch HB, Cooper DS. Management of Graves disease: a review. JAMA. 2015;314(23):2544-54

\section{CONFLITO DE INTERESSES}

Os autores declaram não possuir quaisquer conflitos de interesse.

\section{FINANCIAMENTO}

Os autores declaram não ter recebido qualquer financiamento para o desenvolvimento do estudo.

\section{ENDEREÇO PARA CORRESPONDÊNCIA \\ Sandra Oliveira \\ E-mail: sandrafeoliveira@gmail.com \\ https://orcid.org/0000-0001-5419-163X}

Recebido em 02-06-2017

Aceite para publicação em 03-07-2018

\section{ABSTRACT}

\section{SECONDARY ARTERIAL HYPERTENSION IN A YOUNG ADULT: A CASE REPORT}

Introduction: Secondary arterial hypertension represents about 5-10\% of cases of arterial hypertension. In the presence of hypertension in young adults, secondary causes should be suspected, and the adequate clinical, laboratory and imaging investigations should be performed. In young adults (19-39 years), the most frequent causes of secondary hypertension are thyroid dysfunctions, fibromuscular renal artery dysplasia, and renal parenchymal diseases. We report a case of hypertension in a young woman, whose study revealed an autoimmune hyperthyroidism.

Case report: A 19-year-old woman presented to her family physician with palpitations, tachycardia (heart rate: 117bpm), and high blood pressure (mean BP: $154 / 86 \mathrm{mmHg}$ ) for one month. Anxiety, initial insomnia, irritability, and polyphagia were also present. Physical examination confirmed the presence of high blood pressure values (mean BP: 150/98mmHg) and tachycar$\mathrm{dia}$, as well as palpation of diffuse, painless and symmetrical goiter without apparent nodules. Considering the elevated blood pressure values at two different time points, the diagnosis of arterial hypertension grade I was established, and the patient was medicated with propranolol. Further exams confirmed the diagnosis of autoimmune hyperthyroidism, microcytic and hypochromic anemia, and ultrasound features suggestive of thyroiditis, as well as the presence of diffuse goiter with increased uptake in thyroid scintigraphy, suggestive of Graves' disease. The patient was initially treated with methimazole $5 \mathrm{mg}$ bid, and referred to endocrinology consultation. The treatment resulted in improved symptomatology, tensional stabilization, and normalization of the thyroid function.

Discussion: This case aims to illustrate the diagnostic and therapeutic approach of hypertension in young adults, in a primary care setting. Hyperthyroidism is a common cause of isolated systolic hypertension, and its timely diagnosis and treatment can prevent the occurrence of complications, and influence prognosis. To this end, it is essential to achieve an adequate collaboration between primary and secondary care.

Keywords: Hypertension; Secondary hypertension; Hyperthyroidism; Graves' disease; Young adult 